\title{
A Detailed Comparison of Different Modulations in High Capacity Mode Division Multiplexed Inter-Satellite Optical Wireless Communication System
}

\author{
Amanvir Kaur, Amandeep Kaur Brar
}

\begin{abstract}
Optical wireless communication is getting attention due to edge over radio frequency communication in terms of bandwidth, data rate, security, EMI interference and unlicensed operations. In this work, a mode division multiplexed inter-satellite optical wireless communication is presented at different data rates such as $10 \mathrm{Gbps}, 20 \mathrm{Gbps}$ and 40 Gbps in terms of $Q$ factor. Proposed work is accentuated towards the performance evaluation of modified differential phase shift keying (MD-DQPSK) modulation at different distances $(750 \mathrm{~km}$ to $3750 \mathrm{~km})$. Linear polarized (LP) modes, 64 in number are incorporated in the system to carry the high speed data. Further performance of proposed MD-DQPSK-MDM inter-satellite optical wireless communication is compared with differential quadrature phase shift keying (DQPSK), and Manchester coding and differential phase shift keying (DPSK). Q factor of MDRZDQPSK, DPSK, DQPSK and Manchester coding at $2500 \mathrm{~km}$ of link distance are observed as 16, 8.4, 10.37, and 3.56 respectively at $40 \mathrm{Gbps}$. Results revealed MD-DQPSK provide highest $Q$ factor as compared to DQPSK, DPSK and Manchester modulation.
\end{abstract}

Index Terms-About four key words or phrases in alphabetical order, separated by commas.

\section{INTRODUCTION}

With the progression in the field of optical technology, wireless optical communication is getting popular because of many advantages over radio frequency (RF) communication [1]. Wireless optical communication between satellites is termed as inter satellite optical wireless communication (ISOWC) and this technology has potential to cater high speed data in point to point communication. Laser signals are modulated in ISOWC with data and directed towards receiver through optical wireless channel (typically vacuum) [2]. Long distance transmission and low power operations of optical wireless communication are the prominent benchmarks and it is preferred over microwave technology because it does not pollute the environment. In comparison of free space optical (FSO) and ISOWC, later one is better due to non presence of atmospheric issues such as fog, rain, dust, mist and scintillation effects [3]. In order to boost the capacity of ISOWC systems, wavelength division

Amanvir Kaur, Pursuing M.Tech at Punjabi University, Patiala B.Tech(2013-17) from Punjabi University, Patiala.My research interest is Inter-Satellite Optical Wireless Communication System.

Amandeep Kaur Brar, Assistant Professor at Punjabi University, Patiala from 2012. multiplexing is premier technology where multiple wavelengths operate within single transmission channel incorporating some wavelength spacing. High cost due to need of multiple laser sources and complexity in signal multiplexing/de-multiplexing are two major concerns in WDM system. To wave off the drawbacks of WDM technology, an ultimate technology is suggested by researchers for getting high capacity with only one laser source i.e. Mode division multiplexing (MDM) [4]. In MDM, linearly polarized modes are playing significant role to exploit capabilities of laser signal and these are generated by altering azimuthal as well as radial number of the intensity profile [5]. Suppression of crosstalk by employing Hermitte Gaussian modes in MDM is presented in [6]. Different studies on ISOWC systems are reported such as 32 channel transmission over $5000 \mathrm{~km}$ incorporating DWDM at different input power levels with different modulation formats such as return to zero (RZ) and non return to zero (NRZ) [7]. Investigation of advanced modulation formats was accomplished in [8] at 10,20 and 40 Gbps using modified duobinary RZ, duobinary RZ, carrier suppressed $\mathrm{RZ}$ and it was perceived that at $10 \mathrm{Gbps}, \mathrm{CSRZ}$ and at 40 Gbps, MDRZ found out to be optimal.

In this research article, emphasis is made to generate 64 channels using single laser by employing diverse LG modes using MDRZ-DQPSK, Manchester coding, DPSK, and DQPSK at 10, 20, and 40 Gbps bit rates.

\section{SYSTEM SETUP}

In order to realize proposed model of wireless optical communication between two satellites, a simulation software Optisystem is taken to accomplish the work. Representation of ISOWC system using diverse modulations is depicted in Figure 1 and modulation in this work are MDRZ-DQPSK, Manchester coding, DPSK, and DQPSK. Laser signal is passed through mode generators which can generate different intensity profile (LP modes) by varying the radial and azimuthal numbers. Modulated MDM signals are coupled to the loop control which has 3 essential units such as pre-EDFA (erbium doped fiber amplifier), OWC channel, post-EDFA. Variation in OWC length is carried out from $750 \mathrm{~km}$ to $3750 \mathrm{~km}$ in order to check the effects of distance on proposed system. OWC transmitter/receiver antenna has aperture sizes $15-15 \mathrm{~cm}$. There are $64 \mathrm{LP}$ modes generated and Figure 2 shows the transmitter of 8 LP modes. Distance of OWC is fixed to $250 \mathrm{~km}$ for each loop and distance going to add if loop control value increases. Antenna sizes are 


\section{A Detailed Comparison of Different Modulations in High Capacity Mode Division Multiplexed Inter-Satellite Optical Wireless Communication System}

$15-15 \mathrm{~cm}$ for transmitter and receiver. Different data rates have been considered such as 10, 20 and 40 Gbps with distance variation from $750 \mathrm{~km}$ to $3750 \mathrm{~km}$.

For the generation of LP mode profiles, a CW laser at 193.1 $\mathrm{THz}$ frequency with $10 \mathrm{dBm}$ power is coupled to different 64 mode generations where variation of azimuthal and radial number is performed. These LP mode singles then modulated with modulation as shown in Figure 3 and after communication through transmission loop, these signals are given to mode selectors to lock particular LP mode only. Detection at receiver is performed by single PIN for Manchester coding, MZI-PIN for DPSK to extract 0 and 180 degree phase shifts, MZI-PIN with 45 degree phase delay and time delay is used for decoding of MDRZ-DQPSK signals. After conversion of photons into electric data, suppression of noises is performed by low pass filter and signal regeneration is done (3-R) followed by Eye diagram analyzer to access $\mathrm{Q}$ factors.

Linearly polarized modes are calculated as $M_{N}=n+2 m+1$

Where $\mathrm{m}$ is radial number and azimuthal number is denoted by $\mathrm{n}$

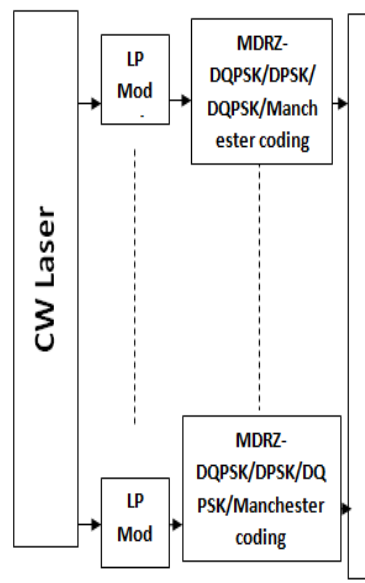

Transmitter Block

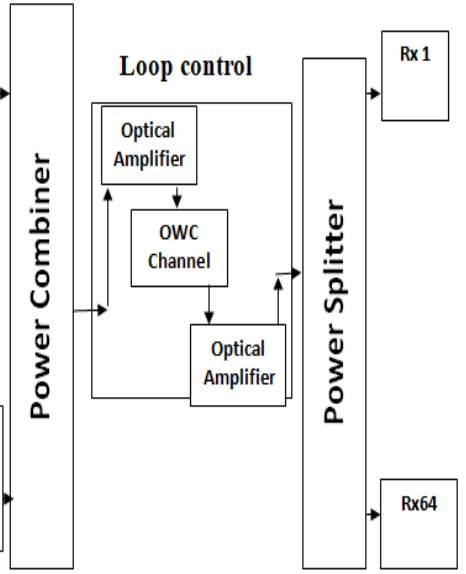

Receiver Block
Fig.1.Proposed ISOWC system for 64 LP modes

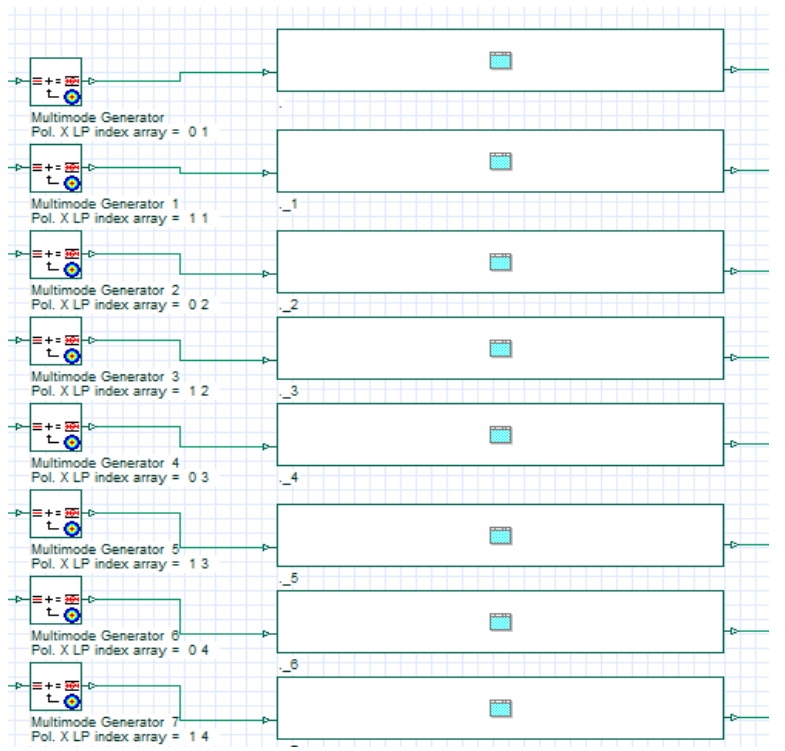

Fig.2.Simulation setup of 8 LP modes
Transmitters of DPSK, DQPSK, Manchester coding, and MDRZ-DQPSK, are represented in Figure 3 (a), Figure 3 (b), Figure 3 (c) and Figure 3 (d) respectively. DQPSK modulation and DPSK has four phase and two phase shifts respectively and these phase shifts increase the dispersion tolerance and spectral efficiency. DQPSK is better than DPSK in terms of endurance to nonlinearities. MDRZ-DQPSK has integrated properties of modified duobinary and differential quadrature phase shift keying which makes it more resistant against chromatic dispersion. In recent years, integrated modulations are getting attention due to their bandwidth efficient spectrum and dispersion tolerance.

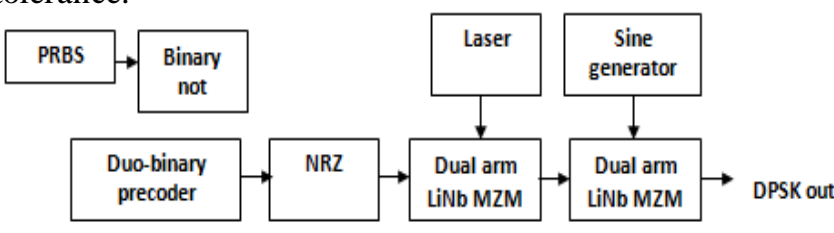

(a)

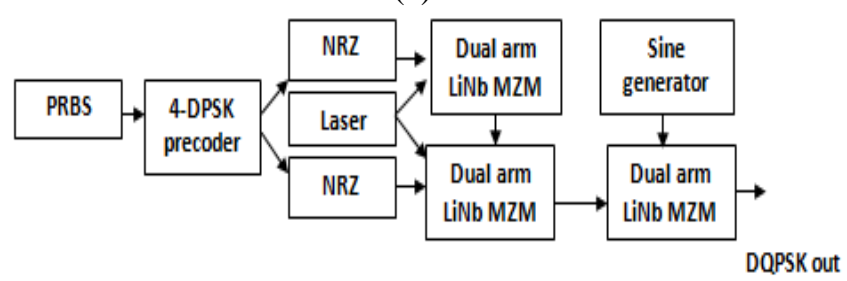

(b)

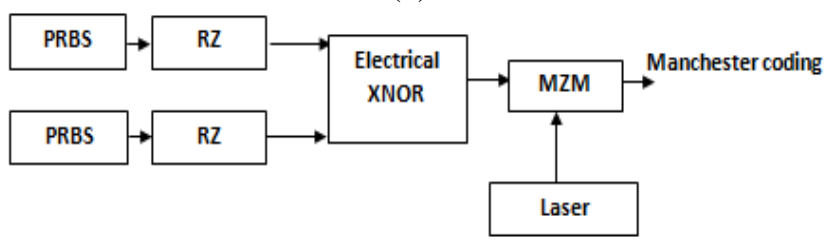

(c)

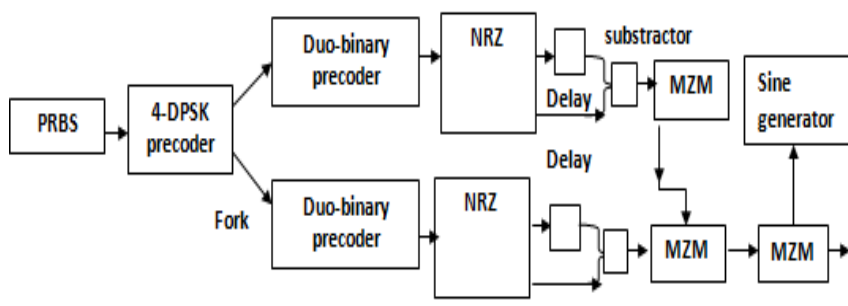

(d)

Fig.3.The schematic of (a) DPSK (b) DQPSK (c) Manchester coding (d) MDRZ-DQPSK

Table I System parameters and values of proposed ISOWC system

\begin{tabular}{|c|c|}
\hline Parameters & Values \\
\hline Data rate & $10,20,40 \mathrm{Gbps}$ \\
\hline Frequency & $193.1 \mathrm{THz}$ \\
\hline Multiplexing & Mode division Multiplexing \\
\hline Modulations/codings & $\begin{array}{c}\text { MDRZ-DQPSK, DQPSK, } \\
\text { DPSK, Manchester }\end{array}$ \\
\hline Number of LP modes & 64 \\
\hline Distance & $750 \mathrm{~km}-3750 \mathrm{~km}$ \\
\hline Power & $30 \mathrm{dBm}$ \\
\hline Amplifiers & EDFA \\
\hline Photo-detector & PIN \\
\hline
\end{tabular}


International Journal of Engineering and Applied Sciences (IJEAS)

ISSN: 2394-3661, Volume-6, Issue-8, August 2019

\section{RESULTS AND DISCUSSION}

To investigate the proposed mode division multiplexing based system, a $64 \times 10 \mathrm{Gbps}, 64 \times 20 \mathrm{Gbps}, 64 \times 40 \mathrm{Gbps}$ inter-satellite optical wireless channels have been simulated in Optisystem software.

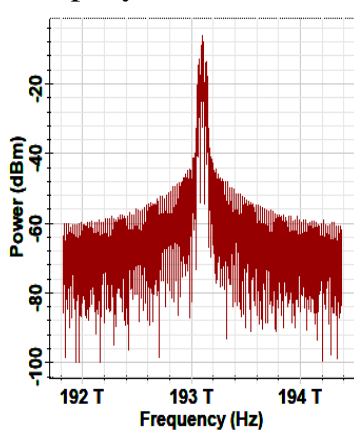

(a)

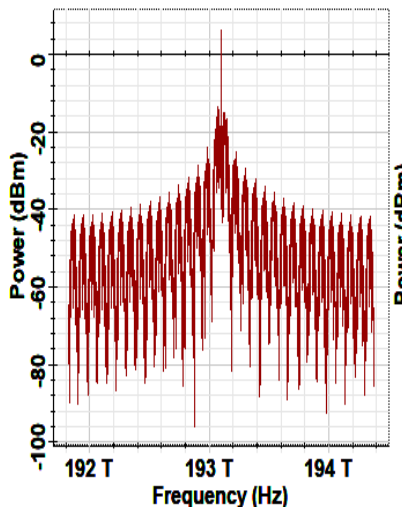

(c)

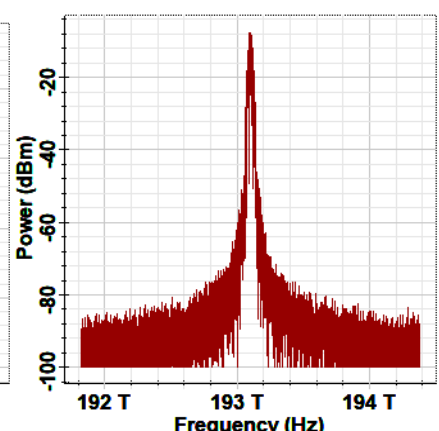

(b)

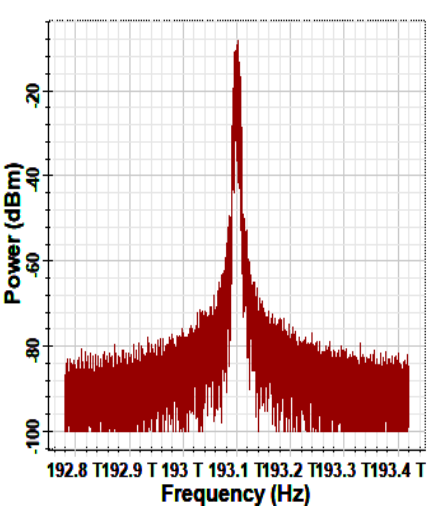

(d)
Fig.4.Optical spectrums of (a) DPSK (b) DQPSK (c) Manchester coding (d) MDRZ-DQPSK

In presented IsOWC system, different modulations and coding has been considered such as MDRZ-DQPSK, DPSK, DQPSK, and Manchester coding. Optical spectrum of differential phase shift keying is depicted in Figure 4 (a), differential quadrature phase shift keying in Figure 4 (b), Manchester coding spectrum in Figure 4 (c) and MDRZ-DQPSK in Figure 4 (d). It is evident that the spectrum of MDRZ-DQPSK is bandwidth efficient and Manchester signal has broad spectrum.

Table 2, depicts the values of $\mathrm{Q}$ factor of different modulations at $10 \mathrm{Gbps}$ and Figure5 (a) depicts the performance of proposed system for different modulations and coding for varied distances at $10 \mathrm{Gbps}$. Distance of each loop is set to be $250 \mathrm{~km}$ and there are different loops considered to provide $750 \mathrm{~km}, 1200 \mathrm{~km}, 2250 \mathrm{~km}, 3000 \mathrm{~km}$ and $3750 \mathrm{~km}$ link length. Figure 5 (a) depicts the performance of different modulation formats at $10 \mathrm{Gbps}$ at varied distances. Results revealed that all the four proposed pulse shapes are in acceptable range of Q factor at 10 Gbps. However, there is reduction $\mathrm{Q}$ as distance prolonged. Results revealed that MDRZ-DQPSK is showing best performance and performance is followed by DPSK till $1200 \mathrm{~km}$ and after that DQPSK surpasses the performance of DPSK. Performance degradation effects of pulse broadening in Manchester coding are severe and therefore its performance is lowest. MDRZ-DQPSK performed best due to is double phase plus quad phase shifting operations which reduces the effects of interference and dispersion. Q factor of
MDRZ-DQPSK, DPSK, DQPSK and Manchester coding at

\begin{tabular}{|c|c|c|c|c|}
\hline $\begin{array}{c}\text { Distance } \\
(\mathbf{k m})\end{array}$ & $\begin{array}{c}\text { MDRZ-D } \\
\text { QPSK }\end{array}$ & DQPSK & DPSK & $\begin{array}{c}\text { Manche-- } \\
\text { ster }\end{array}$ \\
\hline 750 & 78.6523 & 36.4315 & 68.8556 & 9.15678 \\
\hline 1500 & 66.9768 & 31.4902 & 39.5622 & 9.21711 \\
\hline 2250 & 65.4066 & 31.6616 & 31.0824 & 9.22488 \\
\hline 3000 & 59.6172 & 31.6925 & 29.6131 & 9.22628 \\
\hline 3750 & 50.5227 & 31.6988 & 28.1156 & 9.22657 \\
\hline
\end{tabular}

$2500 \mathrm{~km}$ of link distance are observed as 62.26, 29.61, 31.7, and 9.2 respectively. Therefore, use of MDRZ-DQPSK in the inter-satellite mode division multiplexed systems is suggested.

Table II Values of Q factor in different modulations at $10 \mathrm{gbps}$

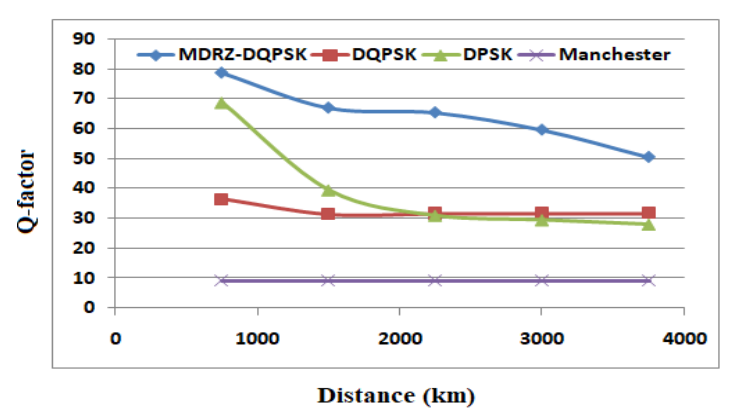

Fig.5.Variation of Q factor with distance of ISOWC channel at $10 \mathrm{Gbps}$

Similarly, Figure 5 (b) depicts the performance of proposed system for different modulations and coding for varied distances at $20 \mathrm{Gbps}$. It is perceived that $\mathrm{Q}$ factor of all different arrangements decreases with distance enhancement as well as all remain within acceptable range of $\mathrm{Q}$ factor.

Distance is varied from $750 \mathrm{~km}$ to $3750 \mathrm{~km}$ at $20 \mathrm{Gbps}$ and it is evident that modified duo-binary return to zero differential quadrature phase shift keying provide highest $\mathrm{Q}$ factor followed by the performance of DQPSK and DPSK with Manchester in the last. MDRZ-DQPSK performed best due to is double phase plus quad phase shifting operations which reduces the effects of interference and dispersion. Q factor of MDRZ-DQPSK, DPSK, DQPSK and Manchester coding at $2500 \mathrm{~km}$ of link distance are observed at $20 \mathrm{Gbps}$ as given in Table 3 .

Table III Values of Q factor in different modulations at 20

\begin{tabular}{|c|c|c|c|c|}
\hline $\begin{array}{c}\text { Distance } \\
(\mathbf{k m})\end{array}$ & MDRZ-DQPSK & DQPSK & DPSK & Manchester \\
\hline 750 & 26.2132 & 27.0021 & 21.1657 & 6.55609 \\
\hline 1500 & 26.4639 & 28.4999 & 19.0376 & 6.61923 \\
\hline 2250 & 25.7839 & 22.0439 & 12.9088 & 6.62742 \\
\hline 3000 & 25.4985 & 22.0582 & 10.8141 & 6.62891 \\
\hline 3750 & 25.2446 & 22.0611 & 8.39581 & 6.62922 \\
\hline
\end{tabular}

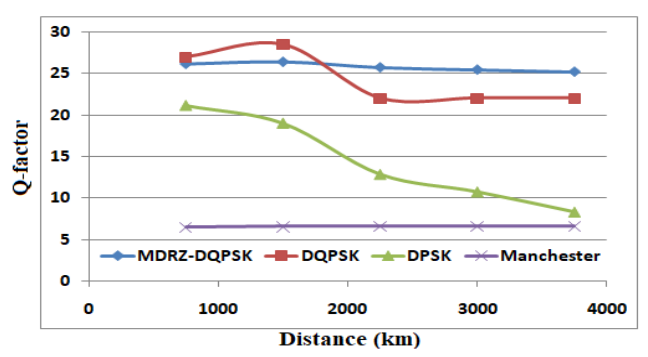


Fig.6. Variation of Q factor with distance of ISOWC channel at $20 \mathrm{Gbps}$

Table IV Values of Q factor in case different modulations at 40 Gbps

\begin{tabular}{|c|c|c|c|c|}
\hline $\begin{array}{c}\text { Distance } \\
(\mathbf{k m})\end{array}$ & MDRZ-DQPSK & DQPSK & DPSK & Manchester \\
\hline 750 & 18.7601 & 16.8808 & 12.7418 & 3.55051 \\
\hline 1500 & 17.9425 & 15.1981 & 10.2749 & 3.62719 \\
\hline 2250 & 16.3580 & 10.3767 & 8.40118 & 3.64178 \\
\hline 3000 & 15.5979 & 9.48992 & 7.63810 & 3.64480 \\
\hline 3750 & 14.8331 & 8.71345 & 6.95984 & 3.64543 \\
\hline
\end{tabular}

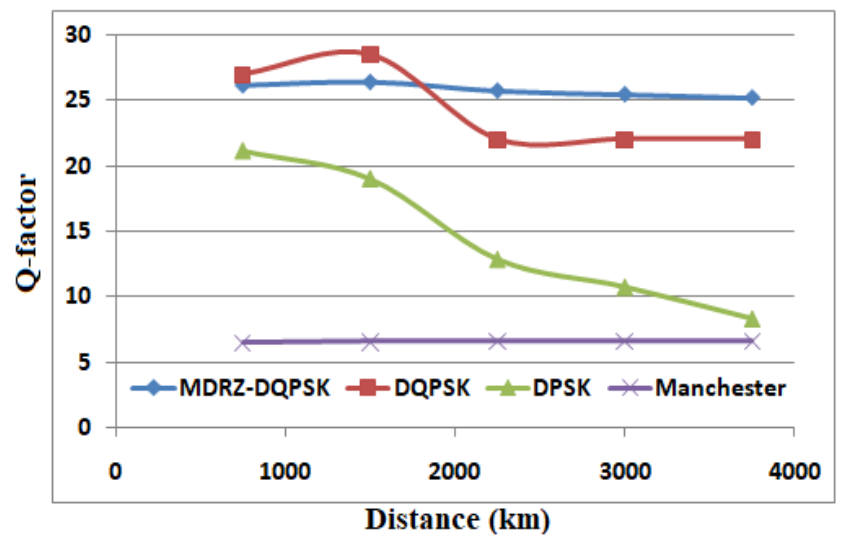

Fig.7. Variation of Q factor with distance of ISOWC channel at $40 \mathrm{Gbps}$

Figure 5 (c) represents the system performance at $40 \mathrm{Gbps}$ for modulations and coding. It is noteworthy that Manchester goes beyond the acceptable range while all the three remaining are in acceptable range. $Q$ factor of MDRZ-DQPSK, DPSK, DQPSK and Manchester coding at $2500 \mathrm{~km}$ of link distance are observed as $16,8.4,10.37$, and 3.56 respectively. Phase shifts in modulations helps system to be resilient against dispersion and nonlinear effects. Performance of Manchester coding is lowest due to absence of phase shifts and due to maximum number of phase shifts, MDRZ-DQPSK system performs optimal.

\section{CONCLUSION}

A 64 channel inter-satellite optical wireless system is investigated for different modulations such as modified duo-binary differential quadrature phase shift keying, differential quadrature phase shift keying, differential phase shift keying and Manchester coding. In order to analyze the effects of data rates, different speeds are considered such as 10, 20 and $40 \mathrm{Gbps}$. Distance of $750 \mathrm{~km}$ to $3750 \mathrm{~km}$ are taken for different data rates and modulations. Major work is to find optimal modulation for IsOWC systems to enhance the reach of the system and to lower the system cost. Q factor of MDRZ- DQPSK, DPSK, DQPSK and Manchester coding at $2500 \mathrm{~km}$ of link distance are observed as 16, 8.4, 10.37, and 3.56 respectively at $40 \mathrm{Gbps}$. It is observed that the MDRZ-DQPSK is best modulation for all data rates and provide maximum $\mathrm{Q}$ and minimum BER.

\section{REFERENCES}

[1] H. Kaur and H. Kaur, " Study of Advanced Intensity and Phase Modulation Formats for IS-OWC DWDM System, Int. J. Eng. Technol.Management Appl. Sci., 5 (2017) 200-208.

[2] Z. Ghassemlooy, S.Rajbhandari and W.Poopola, "Optical Wireless Communications", CRC Press, 2013

[3] A.P. Bindushree, A.B. Nataraju, T.V. Vijesh, A.S. Laxmiprasad, Design and simulation of QPSK modulator for optic inter satellite communication, Int. J.Sci. Technol. Res. 3 (2014) 402-408.

[4]A. Amphawan, S. Chaudhary, T. Elfouly and K. Abualsaud, "Optical mode division multiplexing for secure Ro-FSO WLANs", Adv. Sci. Lett., vol. 21 (2014) 3046-3049.

[5] M. Marken and D.R. Kaur, "Implementation and Analysis of LP modes over OWC link using different pulse shapes',Int. J. Eng. Res.Technol., vol. 4 (2015)261-1265

[6] H. Sarangal, A. Singh,J.Malhotra and S. Chaudhary, "A cost effective 100 Gbps hybrid MDM-OCDMA-FSO transmission system under atmospheric turbulences," Springer Opt. Quant. Electronics, 2017.

[7]P. Kaur, A.Gupta and M. Chowdhary, "Comparative analysis of inter satellite optical Wireless Channel for NRZ and RZ modulation formats for different levels of input power," Int. Symp. Comp. Vision and the Internet, 58 (2015) 572-577.

[8] A. Alipour, A. Mir and A. Sheikhi, "Ultra high capacity inter-satellite optical wireless communication system using different optimized modulation formats,'’Int. J. Light Electron Optics127 (19) (2016) 8135-8143.

Amanvir Kaur, Pursuing M.Tech at Punjabi University, Patiala. B.Tech(2013-17) from Punjabi University, Patiala.My research interest is Inter-Satellite Optical Wireless Communication System.

Amandeep Kaur Brar, Assistant Professor at Punjabi University, Patiala from 2012. 